Revue européenne des sciences sociales

European Journal of Social Sciences

XLV-138 | 2007

Évaluation en sciences sociales. Concepts, mesures et comparaisons

\title{
Les fondements : mesure pour mesure
}

\section{Henri Volken}

\section{(2) OpenEdition}

1 Journals

Édition électronique

URL : http://journals.openedition.org/ress/192

DOI : $10.4000 /$ ress. 192

ISSN : 1663-4446

Éditeur

Librairie Droz

\section{Édition imprimée}

Date de publication : 2 juillet 2007

Pagination : 55-65

ISBN : 2-600-01170-9

ISSN : 0048-8046

Référence électronique

Henri Volken, «Les fondements : mesure pour mesure », Revue européenne des sciences sociales [En ligne], XLV-138 | 2007, mis en ligne le 01 juillet 2010, consulté le 19 avril 2019. URL : http:// journals.openedition.org/ress/192; DOI : 10.4000/ress.192 


\section{Henri VOLKEN}

\section{LES FONDEMENTS : MESURE POUR MESURE}

\section{INTRODUCTION}

Aucun des rituels habituels de l'activité scientifique, dans les domaines des sciences naturelles et des sciences empiriques en tous cas, mais de plus en plus souvent aussi dans les sciences sociales, ne peut se dispenser d'une forme de mesure. Et malgré toute la complexité méthodologique, aussi bien statistique que mathématique et informatique, mise en œuvre pour traiter les informations recueillies, le résultat dépend toujours de manière essentielle de la façon dont celles-ci ont été mesurées. Dès lors de nombreuses questions se posent. Peut-on tout mesurer? Quelle est l'importance du concept quantifiable? Quelles facettes de la réalité méritent le statut de quantité? Est-ce que tout ce qui est quantifiable est également mesurable? De manière générale, l'arithmétique, permettant la manipulation des nombres obtenus dans un processus de quantification, semble sous-jacente à toute activité de mesure.

L'idée de rattacher des nombres à certains attributs des objets qui nous entourent, est omniprésente dans nos activités scientifiques visant à comprendre le monde. En particulier, nous avons l'habitude bien établie de compter des personnes, des choses, des événements et plus généralement tout ce qui apparaît sous forme discontinue, les phénomènes discrets. L'avantage que nous en retirons est la facilité que nous avons de manipuler et de communiquer des nombres et d'avoir ainsi la possibilité de comparer facilement et de manière plus précise les résultats de nos recherches. Mais si nous voulons étendre cette capacité de comparaison numérique à des attributs continus, comme la longueur, ou le poids, ou même le temps, nous sommes amenés à quantifier ces grandeurs, si c'est possible, donc à les diviser en quanta. La mesure est cette opération qui nous fait passer d'un attribut continu, quantifiable, à son expression numérique, nous permettant d'utiliser la simplicité et la flexibilité opérationnelle des nombres pour étendre et transmettre notre savoir sur la réalité.

On peut voir la mesure sous deux aspects différents, représentationnel et pragmatique. Dans le premier cas, elle permet une représentation par des nombres, basée sur des principes scientifiques précis, de la relation entre plusieurs objets par rapport à un attribut choisi. Elle rend donc cette relation plus visible, plus lisible et plus opérationnelle. Dans le second, une certaine forme de mesure, dans un sens plus libre, est introduite dans un dessein spécifique - un indice construit dans un but de comparaison pratique, limitée, par exemple. Ici, c'est la mesure qui crée la variable. Le cas extrême de l'approche pragmatique est probablement justifié par l'opérationnalisme, initié par le physicien Bridgman. Il en 
sera question plus loin. Si ces deux visions de la mesure semblent inconciliables à première vue, il se peut que la plupart des mesures pratiquées, et notamment dans les sciences sociales, contiennent des éléments représentationnels aussi bien que pragmatiques.

\section{ÉTYMOLOGIE ET USAGE}

Un bref coup d'œil sur l'étymologie du concept de mesure peut nous convaincre d'une part de son ancienneté mais aussi de son enracinement dans diverses régions de notre vocabulaire courant et de notre vocabulaire scientifique.

La racine indo-européenne *me, «mesurer», est à l'origine d'une nombreuse famille de mots de notre langue actuelle, dont l'extension montre les divers aspects de la signification de ce concept. Voici un petit échantillon de mots dérivés. Le latin populaire et le latin savant ont produit respectivement «mesure», «démesure», «mesurable» et «immense», « dimension», «commensurable», «mensuration». Le grec est à l'origine de «mètre», «diamètre», «symétrie», «métrologie» et «géométrie ${ }^{1}$. Cette diffusion très large dans notre espace sémantique est un indicateur de l'importance de ce concept dans notre vie courante et, plus particulièrement dans notre activité scientifique. Son caractère ubiquitaire laisse entrevoir également un danger de confusion dans la signification et dans l'emploi de la notion apparemment simple de «mesurer».

Le concept de mesure se retrouve de manière essentielle aussi bien dans la tradition philosophique et littéraire, que dans le domaine des mathématiques et celui des sciences expérimentales et sociales. Voici quelques échantillons de son utilisation: Platon par exemple l'associe à l'ordre cosmique. Il apparaît également avec la signification de «harmonieux », «vertueux», «beau», ou encore «équilibré» chez les philosophes grecs et les théologiens chrétiens, et plus largement dans la tradition philosophique et littéraire traitant de questions d'éthique ou d'esthétique. En mathématiques, la théorie de la mesure s'est développée à propos du problème de la caractérisation du contenu d'un ensemble par un nombre réel. Cette approche a permis le développement de la théorie des probabilités. Mais c'est l'introduction et l'utilisation du concept de mesure dans les sciences expérimentales et sociales qui fera véritablement l'objet de ce texte.

\section{BREF HISTORIQUE}

Si la notion de mesure a joué très tôt déjà un rôle pratique important dans des activités comme l'astronomie, l'architecture et même l'agriculture, les premières formulations explicites et théories cohérentes - dans notre civilisation du moins remontent aux mathématiciens et philosophes grecs à partir de Pythagore et apparaissent de manière plus systématique chez Euclide et Eudoxe. Pour Pythagore et pour son école, tous les rapports de grandeurs sont représentables par des nombres. Cette formulation contient déjà en germe une partie des développements

\footnotetext{
1 Dictionnaire étymologique du français, Jacqueline Picoche, Le Robert 2002.
} 
futurs du concept de mesure. Mais l'existence de grandeurs incommensurables révélée par les pythagoriciens eux-mêmes, qui ont essayé dans un premier temps de cacher cette découverte - est à l'origine d'un effort de clarification de ce qu'est vraiment une mesure. Dans un premier temps, Eudoxe et Archimède ont développé une vision purement géométrique de la mesure, qui se retrouve, en particulier chez Euclide, dans la théorie des proportions, précurseur de la théorie des nombres réels telle que nous la connaissons aujourd'hui. Aristote a fait de la mesure l'une des catégories fondamentales, en distinguant le qualitatif du quantitatif, et dans ce dernier cas, l'aspect discret de l'aspect continu, géométrique. Sa définition ne recouvre que la mesure de grandeurs extensives ${ }^{2}$, c'est-à-dire qui sont révélées par une opération additive concrète.

Il est à remarquer, qu'au XIV ${ }^{\mathrm{e}}$ siècle Oresme soutient la thèse que toutes les variables sont mesurables, qu'elles soient extensives ou intensives ${ }^{3}$. Ce point de vue peut être rapproché de la position opérationnaliste de Stevens - qui ira même plus loin - dans la première moitié du $\mathrm{XX}^{\mathrm{e}}$ siècle. Avec les progrès de la physique on assiste quelques siècles plus tard à une arithmétisation de la mesure, puis l'invention de nouveaux instruments de mesure favorise le développement d'une théorie des mesures physiques. L'efficacité de cette théorie est à l'origine des mesures psycho-physiques et physiologiques introduites par des psychologues comme Fechner en imitation de la démarche dans les sciences naturelles. Les sciences sociales adoptent une approche similaire et c'est la naissance de la sociométrie et de l'économétrie. Ce foisonnement de mesures de nature très diverse dans de nombreux domaines, et les problèmes que cela soulève, sont à l'origine d'une réflexion approfondie sur la notion de quantité et de mesurabilité.

Le vérificationnisme, qui privilégie les conditions de vérification plutôt que la signification d'une énoncé, tend à définir comme mesurables uniquement les grandeurs empiriques, extensives ce qui rend plus difficile, voire impossible, la justification des mesures en sciences sociales et en psychologie. A la suite des travaux de Helmholtz et de Hölder, le physicien Campell jette les bases, en 1920, d'une théorie représentationnelle de la mesure, dans laquelle il distingue les mesures fondamentales et les mesures dérivées. Dans le premier cas, il s'agit des situations où les grandeurs possèdent un ordre et une opération d'addition empiriques, faciles à traduire en termes arithmétiques. Les exemples typiques sont la longueur ou le poids. Mais la théorie de Campbell englobe également des cas plus complexes, possédant une opération empirique indirecte, calculée, comme c'est le cas pour le concept de densité. Campbell parle alors de mesures dérivées.

Cette théorie de la mesure remettait en question les fondements de la démarche des psychologues ${ }^{4}$. Les réactions furent diverses. D'un côté on tendait à ignorer simplement la critique de Campbell ${ }^{5}$, d'autre part, on assistait au développement d'une nouvelle vision, opérationnaliste, de la mesure. Stevens est un représentant radical et influent de cette approche. Pour lui,

2 Dans la terminologie d'aujourd'hui.

3 Voir [6].

4 Surtout après la publication en 1932 du rapport de la British Association for the Advancement of Science.

5 C'est le cas de Bartlett, par exemple. 
une mesure est simplement «l'attribution de nombres à des objets ou événements selon des règles $»$.

La théorie représentationnelle de la mesure, généralement admise aujourd'hui, a été développée de manière systématique conjointement par Krantz, Suppes, Luce et Tversky à partir de 1970, cf [2]. Elle reprend les principales idées de Hölder et Campbell et les généralise dans un système axiomatique rigoureux.

\section{UNE EXPÉRIENCE PROTOTYPIQUE}

L'un des exemples les plus simples et des plus intuitifs d'une expérience de la mesure d'une grandeur continue, est la détermination de la longueur d'un bâton. Supposons que le résultat d'une telle expérience soit exprimé par l'énoncé: «Ce bâton mesure deux mètres ». Les présupposés sémantiques à la compréhension de cet énoncé contiennent au moins les trois éléments suivants:

- l'existence d'un bâton de référence, l'étalon, nommé ici: «mètre»

- une procédure de décision pour savoir si un bâton est plus grand, de même longueur, ou plus petit qu'un autre

- une opération concrète, la concaténation, permettant d' «additionner» deux longueurs et ainsi d'en former une troisième

De cette manière l'énoncé précédent devient compréhensible: «En additionnant deux fois la longueur du mètre, j'obtiens la même longueur que celle du bâton». De même l'énoncé: «Ce bâton mesure un mètre cinquante», signifiera dans une sémantique appropriée: «En additionnant trois fois la longueur du mètre, j'obtiens la même longueur qu'en additionnant deux fois la longueur du bâton ».

L'existence de ces éléments, qui déterminent un ordre et une opération additive, fait d'un attribut d'un objet physique, une quantité. L'attribut devient mesurable. Et le processus de mesure consiste à établir une relation numérique entre deux attributs quantifiables. Cette relation est exprimée par un rapport. Elle implique du point de vue mathématique l'utilisation de nombres rationnels, ou mieux, si l'on veut inclure le cas d'attributs incommensurables, de nombres réels.

\section{LES APPROCHES THÉORIQUES}

Nous allons parcourir très brièvement les principales approches théoriques de la mesure.

La théorie classique de la mesure, décrite systématiquement par Euclide et Aristote, considère exclusivement des attributs extensifs, mais contient de nombreuses idées de la théorie représentationnelle actuelle. Aristote distingue entre une grandeur qui est une multitude si elle est énumérable, et une magnitude si elle est mesurable. Et tous les attributs mesurables sont quantitatifs. Si l'existence de grandeurs incommensurables (côté du carré et sa diagonale par exemple) posait des problèmes aux pythagoriciens, Euclide et Eudoxe ont permis par la théorie des 
proportions $^{6}$ de surmonter cette difficulté, comme nous l'avons vu. Dans cette approche classique ont peut concevoir la mesure comme une procédure d'identification de la relation numérique (proportion) entre une grandeur et une grandeur de référence donnée.

Les débuts de la théorie représentationnelle de la mesure remontent à des considérations du physicien Helmholtz, reprises et systématisées par Hölder et Campbell au début du $\mathrm{XX}^{\mathrm{e}}$ siècle dans une approche axiomatique du concept de quantité. Or cette approche était à la fois rigoureuse et précise, mais - comme nous l'avons vu - trop restrictive pour les utilisations dans les sciences sociales et en psychologie. La vision opérationnaliste de la mesure, dans l'optique de Stevens ${ }^{7}$ en tous cas, se veut une extension de la théorie de Campbell - tout en se libérant de celle-ci - et surtout une vision pragmatique, en ce sens qu'elle définit les concepts scientifiques par les opérations utilisées pour mesurer ceux-ci. Un attribut devient une construction et n'existe que par l'opération de la mesure. Dans cette optique extrême, tout devient mesurable et la recherche de la propriété de quantité n'est plus une priorité.

Entre ces deux positions opposées - celle de Campbell d'une part qui n'admet de mesure que celle qui porte sur une quantité extensive et celle de Stevens qui admet comme mesure toute attribution de nombres à un objet selon une certaine loi - la théorie représentationnelle est considérée maintenant comme la plus généralement acceptée.

L'idée principale de la théorie telle qu'elle nous apparaît aujourd'hui, réside dans la distinction entre système relationnel empirique - la réalité - et système relationnel numérique, et plus particulièrement encore dans le lien qui relie les deux structures. Ce lien est exprimé par la notion d'homomorphisme. Voici quelques détails. Dans la théorie représentationnelle, on considère un ensemble d'objets qui possèdent un ou plusieurs attributs. Prenons le cas d'un attribut unique. Les objets qui ne sont pas distinguables par rapport à cet attribut forment une classe d'équivalence. Les objets, et les relations entre les classes d'équivalence, forment un système relationnel empirique. Nous allons comparer ce système empirique à un système idéal dont les éléments sont des nombres. Et en tant qu'ensemble de nombres, il comporte déjà une relation d'ordre, éventuellement d'autres relations. C'est le système relationnel numérique. Nous allons choisir un ensemble de nombres tels que les relations numériques simulent les relations empiriques. Les correspondants numériques des attributs seront les variables. Le lien recherché entre les systèmes empirique et numérique sera un homomorphisme. Si a est un attribut d'un objet, f(a) est son représentant numérique avec la propriété que si les objets a et b sont dans la relation empirique, alors $\mathrm{f}(\mathrm{a})$ et $\mathrm{f}(\mathrm{b})$ sont liés par la relation numérique. Cela signifie que si un homomorphisme existe entre des systèmes relationnels empirique et numérique, il indique une similarité entre les deux structures. Dans ce cas, la structure numérique imite ou représente la structure empirique. La mesure devient un reflet crédible de l'attribut réel.

$\mathrm{Si}$ en plus de relations sur l'ensemble des objets d'une structure relationnelle empirique, il existe une opération additive concrète, que nous pourrions désigner

\footnotetext{
6 Voir Euclide livre V.

7 A la suite d'idées du physicien Bridgman.
} 
par $\odot$, alors l'homomorphisme f a la propriété suivante $: \mathrm{f}(\mathrm{a} \odot \mathrm{b})=\mathrm{f}(\mathrm{a})+\mathrm{f}(\mathrm{b})$. La mesure respecte le comportement de la structure empirique.

Un exemple: prenons un groupe de personnes et intéressons-nous à leur poids. Les classes d'équivalence sont les sous-groupes de personnes qui ne sont pas distinguables par leur poids. La relation entre ces classes est la relation «être plus lourd que». Le système relationnel empirique est constitué des groupes de personnes de même poids et de cette dernière relation. Un homomorphisme dans ce cas est la fonction qui attribue une valeur numérique, représentant son poids, à chaque personne: $\mathrm{P}(\mathrm{a})$ est le poids de a. Désignons par le symbole $\odot$ l'opération «mettre ensemble sur une balance». Alors $\mathrm{P}(\mathrm{a} \odot \mathrm{b})=\mathrm{P}(\mathrm{a})+\mathrm{P}(\mathrm{b})$ : la mesure $\mathrm{du}$ poids conjoint des deux personnes est la somme des poids individuels.

La structure qui consiste en un système relationnel empirique, un système relationnel numérique et un homomorphisme entre eux, est une échelle de mesure. Mais une échelle de mesure n'est pas toujours unique et même dans la plupart des cas, elle ne l'est pas. Le problème de la représentation consiste à trouver pour un homomorphisme donné, toutes les transformations de cet homomorphisme qui fournissent une échelle de mesure équivalente: $s i \mathrm{f}$ et $\mathrm{g}$ sont des homomorphismes et si $\mathrm{g}=\phi^{\circ} \mathrm{f}$ alors $\mathrm{f}$ et $\mathrm{g}$ sont équivalents. Pour se faire une idée d'un homomorphisme, il suffit de penser à un changement d'unité dans une mesure courante du temps ou de la longueur par exemple.

Les types d'échelles de mesure les plus courantes ${ }^{8}$ sont les suivantes:

- absolue, si $\phi$ est la transformation identité

- de rapport, si $\phi$ est une transformation de similarité: $\phi(x)=a x, a>0$

- d'intervalle, si $\phi$ est une transformation linéaire: $\phi(x)=a x+b, a>0$

- ordinale, si $\phi$ est monotone croissante: si $\mathrm{x}<\mathrm{y}$ alors $\phi(\mathrm{x})<\phi(\mathrm{y})$

- nominale, si $\phi$ est une fonction injective: $\operatorname{si} \phi(\mathrm{x})=\phi(\mathrm{y})$ alors $\mathrm{x}=\mathrm{y}$

\section{LA NOTION DE QUANTITÉ}

La mesure de la longueur d'un bâton nous a permis d'identifier une relation d'ordre et une opération de concaténation qui se trouvent à la base de l'activité de la mesure. Il nous reste à définir tout d'abord ce qu'est une relation d'ordre, puis à énumérer les propriétés que doit remplir une concaténation pour qu'elle puisse donner lieu à une mesure dans le cadre théorique de l'approche représentationnelle.

Nous choisirons de noter $\mathrm{aRb}$ le fait que les objets a et $\mathrm{b}$ soient dans une relation R (par exemple: a est plus grand que b). Une telle relation peut avoir diverses propriétés. Nous en énumérons les principales afin d'être en mesure de définir les concepts d'ordre et d'équivalence:

- Réflexivité:

$\mathrm{aRa}$

- Symétrie:

$\mathrm{Si} \mathrm{aRb}$, alors bRa

8 Voir [8]. 
- Transitivité:

- Antisymétrie:

- Asymétrie:

- Transitivité négative:

- Complétude:

- Complétude forte:
$\mathrm{Si}$ aRb et bRc, alors aRc

$\mathrm{Si}$ aRb et $b \mathrm{Ra}$, alors $\mathrm{a}=\mathrm{b}$

$\mathrm{Si} \mathrm{aRb}$, alors non bRa

Si non $\mathrm{aRb}$ et non bRc, alors non aRc

Si $\mathrm{a} \neq \mathrm{b}$, alors $\mathrm{aRb}$ ou $\mathrm{bRa}$

$\mathrm{aRb}$ ou bRa

Sans une relation d'ordre, aucune mesure digne de ce nom ne peut être définie. Une relation d'ordre est un concept très intuitif, omniprésent dans la vie scientifique et quotidienne. Néanmoins, dans un effort de fonder la notion de mesure de manière rigoureuse, il est indispensable de distinguer les nuances suivantes:

- ordre partiel:

- ordre faible

- ordre faible strict:

- ordre simple strict: la relation est réflexive, antisymétrique et transitive la relation est transitive et fortement complète la relation est asymétrique et négativement transitive la relation est transitive, asymétrique et complète

Cette terminologie permet de voir, qu'en parlant de bâtons, la relation «a est de même longueur ou plus long que $b$ » est un ordre faible, alors que la relation « $\mathrm{a}$ est plus long que $\mathrm{b}$ » est un ordre faible strict. Si par contre dans un certain contexte, deux objets ne sont pas comparables dans une relation d'ordre, celle-ci sera partielle. Ça peut être le cas dans une comparaison multi-critère.

Le point crucial est le théorème de Birkhoff-Milgram qui donne les conditions nécessaires et suffisantes pour que les objets sur lesquels porte une relation empirique $\mathrm{R}$, un ordre faible strict, puissent être mis en rapport avec des nombres de manière à satisfaire la condition: $a R b$ si et seulement si $f(a)>f(b)$, où $f(a)$ et $f(b)$ sont les représentations numériques des objets a et $\mathrm{b}$. Ces représentations sont appelées des variables. Les deux conditions données par Birkhoff-Milgram sont essentiellement que la relation $\mathrm{R}$ soit un ordre faible strict et qu'elle possède la propriété de densité suivante: $\mathrm{Si} \mathrm{aRb}$, alors il existe un élément c tel que aRc et cRb.

Le problème de la mesure peut être posé de la manière suivante: étant donné un ensemble d'objets $A$, une relation empirique $\mathrm{R}$ et une opération de concaténation, à quelle condition peut-on trouver une fonction $\mathrm{f}$ qui représente les objets par des nombres réels, une relation > sur les nombres et une opération arithmétique + , telles que:

- $a R b$ si et seulement si $f(a)>f(b)$, et

- $f(a, b)=f(a)+f(b)$

Dans ce cas, f(a) sera la mesure de a selon l'attribut choisi. Plusieurs systèmes axiomatiques ont été proposés pour résoudre le problème de la mesure, notamment celui de Hölder (1901). Le système qui suit ([3]), est à la fois nécessaire et suffisant, ce qui n'était pas le cas du système de Hölder. Les quatre axiomes de ce système sont les suivants: 
- La relation $\mathrm{R}$ est un ordre faible strict

- Elle est faiblement associative, ce qui signifie que les deux objets construits a. (b.c) et (a. b). c sont équivalents: ils ne sont pas dans la relation R, ni dans une direction ni dans l'autre

- R est une relation monotone, c'est-à-dire que: $\mathrm{aRb}$ si et seulement si, pour tout objet c, on a (a. c)R(b. c) et aussi (c. a)R(c. b)

- R est une relation archimédienne, autrement dit, pour tous les objets a,b,c,d: si $\mathrm{aRb}$, alors il existe un nombre $\mathrm{n}$ tel que (na. c) $\mathrm{R}(\mathrm{nb}, \mathrm{d}$ )

Le dernier axiome signifie que quelle que soit la relation entre $\mathrm{c}$ et $\mathrm{d}$, il existe toujours un nombre $\mathrm{n}$ de concaténations de $\mathrm{a}$ et de $\mathrm{b}$ qui rétablit la relation $\mathrm{R}$ entre (na. c) et (nb d). Par exemple, si l'attribut d'un objet est son poids, et que . représente l'opération de placer deux objets ensemble sur une balance, la propriété archimédienne signifie: si a est plus lourd que $b$, alors quel que soit le poids des objets c et d, un certain nombre de copies de a plus c sera toujours plus lourd que ce même nombre de b plus d.

Les attributs des objets d'une telle structure sont des quantités et sont donc mesurables. Les quatre axiomes garantissent que pour deux quantités a et b, la grandeur de a peut toujours être exprimée par un nombre réel $r$, tel que $a=r b$. La mesure extensive peut donc être définie simplement:

- La mesure est l'estimation ou la découverte du rapport d'une grandeur par rapport à une unité du même attribut.

La mesure extensive dépend de la découverte d'une relation d'ordre et d'une opération de concaténation qui reflète directement, physiquement, la structure quantitative de l'attribut impliqué. Elle joue un rôle très important dans les sciences physiques et naturelles, mais son application dans les sciences sociales est beaucoup plus difficile, ce qui est dû en partie à la nature des objets étudiés et aux instruments pour en appréhender les attributs. D'autre part, cette mesure ne porte que sur un attribut isolé. Dans les sciences sociales, l'intérêt se porte souvent sur l'interaction de différents attributs, qui n'ont pas toujours les propriétés requises par les définitions précédentes. L'analyse conjointe que nous allons présenter brièvement, est particulièrement adaptée à la méthodologie des sciences sociales.

\section{LA MESURE CONJOINTE}

Le but de cette méthode est de révéler la nature quantitative, donc mesurable, d'une structure qui relève a priori uniquement de la relation d'ordre et qui relie plusieurs variables. Cette situation se rencontre couramment dans les sciences sociales. C'est le cas par exemple lorsque nous considérons un bien de consommation selon le degré de satisfaction qu'il procure, satisfaction qui peut dépendre de plusieurs facteurs comme le prix, la qualité et autres. La satisfaction peut être ordonnée sur une échelle simple, les autres caractéristiques peuvent être catégorielles ou numériques. Nous allons présenter - sans entrer dans les détails 
trop techniques - le cas le plus simple où une variable ordonnée dépend de deux autres facteurs ${ }^{9}$.

Plus précisément, considérons la variable A qui représente numériquement un attribut, et qui dépend de deux facteurs B et C. Numériquement, A est décrite par une fonction $\mathrm{f}$, qui fait correspondre à un attribut de $\mathrm{B}$ et un attribut de $\mathrm{C}$ une valeur numérique de A. Le but de la mesure conjointe est de représenter la fonction $\mathrm{f}$, si ses images forment un ordre faible, par une somme de fonctions $\mathrm{g}$ et $\mathrm{h}$ sur les attributs $B$ et $C: f(b, c)=g(b)+h(c)$. Si cela réussit, il aura donc été possible de dériver des échelles additives sur $\mathrm{B}$ et $\mathrm{C}$ à partir de la relation d'ordre faible sur $\mathrm{A}$. On peut montrer que dans ce cas, $g$ et h déterminent des échelles d'intervalle dans la classification de Stevens. Autrement dit, on aura établi la nature quantitative des variables dont a priori on ne perçoit pas très bien la structure mesurable.

Il nous reste à dire dans quelles conditions cette structure additive conjointe existe. Luce et Tukey (voir [1]) ont établi une liste de conditions pour cela:

- A doit correspondre à un ordre faible $\mathrm{R}$

- R satisfait la condition de solvabilité: Pour toutes les valeurs $\mathrm{a}_{1}$ dans $\mathrm{A}$, et toutes les valeurs $b_{1}, b_{2}$ dans $B$, il existe une valeur $a_{2}$ dans $A$ telle que $\left(a_{1}, b_{1}\right)$ $R\left(a_{2}, b_{2}\right)$ et aussi $\left(a_{2}, b_{2}\right) R\left(a_{1}, b_{1}\right)$. Inversement pour toutes les valeurs $a_{1}, a_{2}$ dans $A$ et $b_{1}$ dans $B$, il existe une valeur $b_{2}$ dans $B$ telle que $\left(a_{1}, b_{1}\right) R\left(a_{2}, b_{2}\right)$ et $\left(a_{2}, b_{2}\right) R\left(a_{1}, b_{1}\right)$

- La condition de double annulation doit être satisfaite: pour toutes les valeurs $a_{1}, a_{2}, a_{3}$ de $A$ et toutes les valeurs $b_{1}, b_{2}, b_{3}$ de $B$, la conditionnelle suivante doit toujours être vérifiée: $\operatorname{Si}\left(a_{2}, b_{1}\right)$ R $\left(a_{1}, b_{2}\right)$ et $\left(a_{3}, b_{2}\right) R\left(a_{2}, b_{3}\right)$ alors $\left(a_{3}, b_{1}\right)$ $\mathrm{R}\left(\mathrm{a}_{1}, \mathrm{~b}_{3}\right)$

- Toute suite standard de valeurs strictement bornée, aussi bien dans la première que dans la deuxième composante, est finie ${ }^{10}$

Cette dernière condition est plus technique mais signifie essentiellement qu'aucune différence entre éléments de $\mathrm{B}$ ou de C n'est infiniment plus grande qu'une autre différence de ce type.

Le résultat essentiel pour les sciences sociales est le suivant: Sous ces quatre conditions, on peut affirmer la mesurabilité additive des attributs $B$ et $C$, ce qui constitue une généralisation et un assouplissement de la notion de quantité et de mesure [1].

\section{MESURE DE L'INCERTITUDE: LA THÉORIE DES PROBABILITÉS}

Les sciences empiriques procèdent de préférence par induction. Mais cette démarche exige une notion de «vraisemblance», de «probabilité » dans un sens

9 Il y a de nombreuses généralisations. Par exemple en considérant plus de deux facteurs ou encore en ne se bornant pas à trouver une représentation additive de $\mathrm{f}$.

${ }^{10}$ Nous ne donnerons pas ici la définition de suite standard. Voir pour cela [6] ou [9] 
large, capable d'étayer la théorie qui en résulte, à défaut de la justifier. La relation «l'événement A n'est pas plus probable que l'événement B» définit une relation d'ordre faible sur les événements que nous observons. Si nous arrivons à définir une notion de mesure de cette probabilité, nous aurons à disposition une théorie capable d'organiser et de structurer efficacement notre savoir. Les difficultés pour y arriver sont d'ordre formel et épistémologique. Commençons par le problème formel. Comment mesurer un objet mathématique?

Mathématiquement il y a deux façons de mesurer un ensemble, l'objet de base le plus répandu des mathématiques. Si cet ensemble est constitué d'éléments individuels, séparés, discrets, on peut mesurer sa taille en "comptant» les éléments. Nous avons pour cela à disposition la théorie des nombres cardinaux, qui constitue une modélisation de la notion de dénombrement et qui la généralise dans le cas infini. Mais les objets géométriques, les objets continus, ont largement échappé à cette approche. Comment mesurer une surface, un volume par exemple? Une théorie mathématique différente s'est développée dès le XIX ${ }^{\mathrm{e}}$ siècle, dont l'objectif est non pas de mesurer la taille, mais le contenu d'un ensemble. Elle tente d'établir une mesure numérique pour le plus grand nombre possible de sous-ensembles d'un ensemble. Si l'on exige que cette mesure soit additive et invariante par rapport à une isométrie, c'est-à-dire une transformation qui laisse invariantes les distances, on peut montrer qu'une telle mesure n'existe que sous certaines conditions. En particulier elle n'existe pas pour l'ensemble de tous les sous-ensembles. Il faut par conséquent se limiter à des classes restreintes de sous-ensembles. C'est à E. Borel que l'on doit d'avoir introduit la notion de tribu, qui permet de définir une telle mesure. Voici quelques définitions qui illustrent le cadre formel dans lequel une mesure peut exister.

Un ensemble de sous-ensembles de E est une tribu, si les conditions suivantes sont remplies:

- l'ensemble vide, en fait partie

- avec chaque ensemble, son complément en fait aussi partie

- si une famille dénombrable de sous-ensembles en fait partie, alors la réunion en fait partie aussi

Sur une tribu, définie sur un ensemble, il existe une mesure $\mu$ si les conditions suivantes sont remplies:

- l'ensemble vide est de mesure 0

- la mesure d'une réunion dénombrable d'ensembles est la somme des mesures individuelles

Des résultats de Lebesgue établissent des théorèmes d'existence de mesures sur une tribu, rendant possible diverses théories mathématiques centrées sur un processus de mesure, dont la théorie des probabilités est l'un des exemples importants. En particulier pour les sciences expérimentales. Ce que nous aimerions mesurer dans ce dernier cas, afin de réduire l'incertitude, c'est la relation empirique «tel événement est au moins aussi probable que tel autre».

Le concept de mesure de probabilité est lui-même objet de débat depuis plus d'un siècle. Faut-il considérer la probabilité d'un événement comme un indice 
représentant notre croyance subjective, comme le soutient l'approche subjective, bayesienne, ou, et c'est le point de vue fréquentiste, comme un nombre idéal vers lequel tendrait la fréquence de l'occurrence de cet événement. Le problème n'a pas été résolu, mais la discussion autour de cette question s'est faite plus discrète. Peut-être parce que la théorie mathématique sous-jacente à ces deux écoles est la même. Il s'agit essentiellement de la théorie que nous venons d'évoquer, avec la particularité que dans le cas de la théorie de la probabilité la mesure est normée, c'est-à-dire que la mesure de l'ensemble E est fixée à 1. L'ensemble E, l'ensemble fondamental, regroupe tous les résultats possibles d'une expérience aléatoire, et les événements forment une tribu sur E. Cette tribu est choisie en fonction de critères pragmatiques, parmi lesquels figurent les suivantes:

- quelle est la précision du langage que nous voulons adopter? Autrement dit, avec quel degré de détails voulons nous parler des résultats de l'expérience?

- quelle est l'utilisation que nous voulons faire de la notion de probabilité?

Un petit exemple permet d'illustrer ce point. Le dé à jouer produit une expérience aléatoire particulièrement simple et courante. Les résultats peuvent être représentés par l'ensemble $E=\{1,2,3,4,5,6\}$. Si nous ne sommes intéressés que par la parité du résultat, nous pouvons choisir la tribu constituée par l'ensemble vide et les sous-ensembles $\{1,3,5\},\{2,4,6\}$ et $\{1,2,3,4,5,6\}$, c'est-à-dire que nous distinguons les événements: impossible, impair, pair et certain. Mais si nous voulons distinguer tous les résultats, nous choisirons la tribu qui contient tous les 64 sous-ensembles de E. Par exemple $\{1,5,6\}$ représentera l'événement «le résultat est plus petit que 2 ou plus grand que $4 »$. Dans les deux cas, nous pouvons choisir comme mesure d'un événement simplement son cardinal - c'est-à-dire le nombre de résultats qu'il contient - divisé par le cardinal de E. C'est le modèle équiprobable habituel.

La théorie mathématique des probabilités, basée sur l'axiomatisation de Kolmogorov, offre un cadre formel dans lequel les calculs se font essentiellement de la même manière, que l'on se fonde sur l'approche subjectiviste ou fréquentiste. Mais la méthodologie se différencie fortement selon les présupposés conceptuels concernant la notion de la mesure de la probabilité. Dans de nombreuses situations les calculs se ramènent au modèle équiprobable dont il a été question ci dessus. C'est le cas lorsque dans une collectivité on cherche à se faire une idée de la « chance» qu'un objet de cette collectivité ait une certaine propriété. L'approche fréquentiste est alors évidente puisque dans ce modèle on choisit une notion de proportion pour représenter la probabilité. D'autres formes de représentations de «probabilité» sont plus ouvertement subjectives. L'occurrence redoutée d'une catastrophe dans un certain contexte ne peut être traitée de la même manière, puisque l'ensemble fondamental n'est pas clairement défini. Des éléments plus subjectifs, ou provenant d'autres sources sont alors intégrés dans l'établissement d'un indice de probabilité. Mais quelle que soit l'approche, la partie mathématique se base ensuite sur l'axiomatique de Kolmogorov. 


\section{MESURE DANS LES SCIENCES SOCIALES}

Dans les sciences physiques, les objets pris en considération, sont la plupart du temps individuels, discrets. C'est le cas classique de l'atome, du point masse ou du mobile se déplaçant dans un champ de force. Et toute l'attention de la physique se porte sur ces individualités: les équations décrivent le comportement de ces objets distincts, en fonction de leurs attributs. Or dans les sciences sociales, la situation est tout autre. Ici les objets sont souvent de nature collective, agrégée. Les propriétés d'un tel ensemble ne sont plus simplement la somme des propriétés des individus qui le constituent, mais peuvent être, le plus souvent, des émergences, des qualités qui sont propres à l'ensemble et qui n'apparaissent pas chez aucun de ces individus. Les concepts de société, de population et de la manière de les appréhender, notamment par les notions de taux ou de différentes moyennes ou autres descripteurs statistiques, sont relativement tardifs et à l'origine de nombreux débats épistémologiques. Il suffit de penser, par exemple, à la signification du taux de criminalité et de l'évolution de celle-ci dans un contexte urbain. La régularité et la périodicité de la criminalité ne peuvent pas être imputées à des individus, mais à des unités d'une autre nature, des collectivités. Le concept de $\operatorname{taux}^{11}$, si fréquent dans les discussions sociologiques depuis le XIX ${ }^{\mathrm{e}}$ siècle, est une construction statistique et ne reflète pas non plus des propriétés individuelles. Un autre exemple célèbre est fourni par la querelle animée autour de la notion de «l'homme moyen» telle qu'elle apparaît par exemple chez Quetelet, puis dans les discussions autour de ce que l'on appelait au XIX ${ }^{\mathrm{e}}$ siècle la statistique sociale.

Dans ce contexte, la mesure possède une forte connotation pragmatique. Les sciences sociales, dont le but est de comprendre et décrire la manière dont les personnes interagissent en groupes, utilisent des concepts globaux comme les attitudes, les motivations, les préférences, ou dans le domaine économique, de production, de consommation, d'inflation. Ces concepts ne sont pas mesurables directement et nécessitent une construction théorique pour les approcher empiriquement. Cela peut se faire, dans les meilleurs cas, rigoureusement, mais au prix d'un effort souvent considérable, par la méthodologie de la mesure conjointe. Quoi qu'il en soit, cela demande toujours l'établissement d'un ensemble de conventions si l'on veut partager et communiquer les résultats. Dans toutes ces situations, il n'est pas toujours très clair ce que l'on mesure exactement, et si ce que l'on mesure est ce qu'on prétend étudier. Très souvent les sciences sociales, mais aussi la psychologie, semblent se trouver dans la situation où c'est précisément la mesure qui crée le concept. Cet aspect pragmatique est la conséquence de la complexité inhérente aux objets de ces domaines, mais aussi de la portée sociale et politique que peuvent y avoir les résultats des recherches.

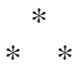

On peut retenir de cette brève présentation de la mesure, qu'il existe une notion précise de ce qu'est une mesure. Cette notion se base sur l'idée de quantité

${ }^{11}$ Et les nombreux indices introduits notamment en économie. 
et sur la certitude que ce sont les attributs quantifiables qui sont mesurables dans un sens strict. Il existe des critères précis de ce qu'est une quantité. La vérification empirique de ces critères devrait donc être une activité préliminaire à toute mesure. Or cette approche rigoureuse n'est possible que dans certains cas et se limite aux sciences exactes. Et même en physique, si la longueur, le poids sont des attributs directement mesurables, la densité par exemple est une grandeur qui n'est mesurable qu'indirectement. Les variables extensives, pour lesquelles il existe une opération empirique additive explicite, ne sont qu'une minorité. Une extension de la notion de mesure à des variables intensives s'impose. L'approche représentationnelle actuelle fournit un cadre rigoureux, permettant néanmoins la mesure de grandeurs plus générales.

Les sciences sociales cependant se sont affranchies d'avantage encore de ce cadre, en particulier à cause des concepts complexes, collectifs qui les sous-tendent. Mais également par l'aspect pragmatique que prend la plupart du temps l'activité de mesure. Les attributs construits sont souvent l'expression d'une démarche spécifique, autour d'un problème localisé dont l'intérêt n'est pas purement scientifique, mais comporte des retombées dans d'autres domaines, politiques, économiques, environnementaux ou culturels. La composante pragmatique de la mesure dans la méthodologie des sciences sociales s'illustre clairement dans le fait que les attributs mesurés ne sont finalement définis, la plupart du temps, que par le processus de la mesure.

\section{Université de Lausanne}

\section{RÉFÉRENCES}

[1] Krantz, D.H., 1964, Conjoint measurement : the Luce-Tukey axiomatisation and some extensions, J. Math. Psy. 1

[2] Krantz, D.H., R.D. Luce, P. Suppes \& A. Tversky, 1971, Foundations of measurement, vol. I, Academic Press

[3] Hand, D.J., 2004, Measurement. Theory and pratice, Arnold

[4] Luce, R.D., The ongoing dialog between empirical science and measurement, J. Math. Psy. 40

[5] Michell, J., 1994, Numbers as quantitative relations and the traditional theory of measurement, Brit. J. Phil. Sci. $45: 2$

[6] Michell, J., 1999, Measurement in psychology, Cambridge University Press

[7] Scott, D. \& P. Suppes, 1958, Foundational aspects of theories of measurement, J. of Symb. Logic, vol 23

[8] Stevens, S.S., 1946, On the theory of scales of measurement, Science, 103

[9] Suppes, P. \& J. Zinnes, 1963, Basic measurement theory, in: Handbook of Mathematical Psychology, vol. I, Wiley 